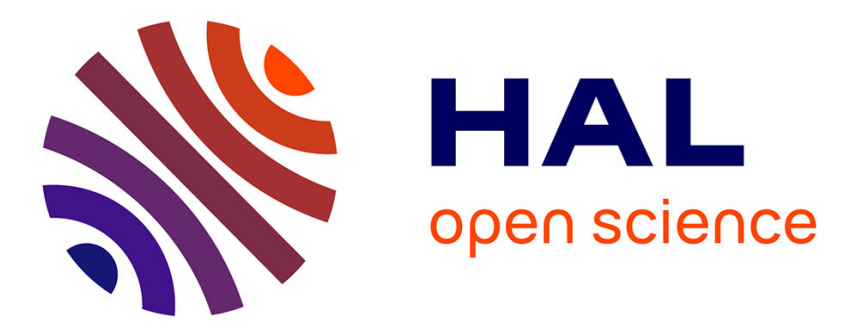

\title{
Constructions transitives et causative attributive du verbe "faire"
}

Takuya Nakamura

\section{To cite this version:}

Takuya Nakamura. Constructions transitives et causative attributive du verbe "faire". K. Ogata. Autour des verbes: constructions et interprétations, 29, John Benjamins, pp.59-86, 2013, Lingvisticae Investigationes Supplementa. hal-01586029

\section{HAL Id: hal-01586029 https://hal.science/hal-01586029}

Submitted on 12 Sep 2017

HAL is a multi-disciplinary open access archive for the deposit and dissemination of scientific research documents, whether they are published or not. The documents may come from teaching and research institutions in France or abroad, or from public or private research centers.
L'archive ouverte pluridisciplinaire HAL, est destinée au dépôt et à la diffusion de documents scientifiques de niveau recherche, publiés ou non, émanant des établissements d'enseignement et de recherche français ou étrangers, des laboratoires publics ou privés. 


\title{
Constructions transitives et causative attributive du ve rbe faire
}

Takuya Nakamura

Université Paris-Est, LIGM (UMR 8049 CNRS)

\begin{abstract}
French sentence structure $N$ faire de $N N$ is shown to present a three-way ambiguity, according to the use of the verb faire: ordinary transitive and support (or light) faire constitute a transitive structure, but the third one, "causative operator faire", makes a peculiar one in which a direct nominal $(N)$ functions, not as a direct object, but as a indirect object $(d e N)$ complement. As an operator, the verb takes as "operand" a particular type of copular sentence, i. e. identificational type, on the contrary to rendre and mettre, well-known causative verbs, applied only to predicational type. faire, rendre and mettre are in complementary distribution. Different possibilities of causative operators for different types of copular sentences are also discussed.
\end{abstract}

\section{Introduction}

Dans la première partie de cette étude, nous montrons qu'une même forme de construction syntaxique peut être fonctionnellement différenciée de trois façons (§2). Il s'agit de la structure $N V$ de $N N$, dont le verbe principal est le verbe faire. On est amené à y distinguer trois types de constructions syntaxiques sur la base de la différence des fonctions grammaticales que jouent les deux compléments post-verbaux de $N$ et $N$ dans les phrases et d'une restriction sémantique sur le sujet. Parmi ces trois types, nous identifions une construction particulière où la relation entre les deux compléments post-verbaux est celle de prédication et où c'est le $N$ directement régi par le verbe qui sert de prédicat par rapport à (de) $N$. C'est une construction à attribut de l'objet ind irect.

Dans la seconde partie $(\$ 3)$, pour caractériser cette prédication imbriquée dans la construction du verbe faire, nous la comparons à la typologie des phrases copulatives. Il résulte de cette comparaison que ce verbe peut s'analyser comme un opérateur causatif appliqué à un type de phrases copulatives. Il est de ce point de vue en distribution complémentaire avec rendre et mettre.

Les opérateurs causatifs rendre, mettre et faire ne s'appliquent pas, toutefois, à tous les types de phrases copulatives. On examinera si les trous restant dans la distribution des opérateurs causatifs peuvent être comblés par d'autres constructions du verbe faire et, si ce n'est pas possible, par d'autres constructions d'autres verbes. 


\section{Distinction de tro is types de construction syntaxique}

Voici les trois types de phrase qui font l'objet, dans ce qui suit, de différenciation fonctionnelle, constituées chacune d'un sujet, d'un complément prépositionnel et d'un complément direct :
A. Paul a fait de ce bois une belle table
B. Paul a fait de ce type de bois son matériau de prédilection
C. Paul a fait de ce type de bois une utilisation intéressante

Les trois types de phrase présentent à la surface une même structure, $N V$ de $N N$, sur le même verbe faire. Dans ce qui suit, nous énumérons pour chaque constituant les traits syntaxiques et sémantiques dont la configuration particulière différencie chaque type de phrase des autres. Les trois types A, B et C correspondent à des emplois différents du verbe faire.

\subsection{Sujet}

Seuls les sujets des types $\mathrm{A}$ et $\mathrm{C}^{1}$ ont une restriction sur l'interprétation : ils doivent être agentifs. Le sujet du type B peut ne pas l'être :

(1) (Paul, *son outil) a fait de ce bois une belle table

(2) (Paul, son penchant pour le postmoderne) a fait de ce type de bois son matériau de prédilection

$$
\text { (Paul, *son habileté) a fait de ce type de bois une utilisation intéressante }
$$

\subsection{Comportements du complément en de}

Tous les compléments en de partagent les propriétés formelles et sémantiques suivantes : (a) la cliticisation par en ; (b) l'interrogation par de quoi ; (c) la relativisation par dont; (d) pas de restriction sur la définitude ; (e) le référent du $S N$ régime peut être humain ou non humain.

Voici les résultats de l'application des tests (a)-(e) au type A :

(4) a. Paul en a fait une belle table, de ce bois

b. De quoi Paul a-t-il fait une belle table ? - De ce bois

c. (...) ce bois dont Paul a fait une belle table (...)

d. Paul a fait de (un morceau de, ce) bois une belle table

e. Le président a fait de ces 34 ministres un nouveau gouvernement

Les exemples (5) montrent les résultats de l'application des mêmes tests au type B :

(5) a. Paul en a fait son matériau de prédilection, de ce type de bois 

b. De quoi Paul a-t-il fait son matériau de prédilection? - De ce type de bois
c. (...) ce type de bois dont Paul a fait son matériau de prédilection (...)
d. «Elles racontent, maquettes et plans à l'appui, le développement qui a fait d'un château fort le «plus grand musée du monde » et disent, sobrement et clairement, l'essentiel de ce que dirait un guide compétent $\gg$ (Le Monde, 1994)
e. Qu'est-ce qui fait de cet enfant un tyran?

Voici, cette fois, les résultats de l'application des mêmes tests au type C :

(6) a. Paul en a fait une utilisation intéressante, de ce type de bois

b. De quoi Paul a-t-il fait une utilisation intéressante ? - De ce type de bois

c. (...) ce morceau de bois dont Paul a fait une utilisation intéressante (...)

d. Paul a fait de (un, ce) morceau de bois une utilisation intéressante

e. Paul a fait de ces jeunes acteurs une utilisation intéressante

Les résultats de l'application des tests (a)-(e) aux trois types montrent clairement que le complément en de fonctionne comme un complément du verbe dans les trois types.

Il existe toutefois deux propriétés qui distinguent une construction des deux autres : (f) la possibilité de ne pas se construire avec ce complément ; $(\mathrm{g})$ la substitution de ce complément par un autre complément prépositionnel (par exemple, avec $S N$, à partir de $S N)$. En $(7 \mathrm{f})-(7 \mathrm{~g})$, nous donnons les résultats de l'application de ces deux tests au type A :

(7) f. Paul a fait une belle table

g. Paul a fait (avec, à partir de) ce bois une belle table

Les exemples (8f)-(8g) et (9f)-(9g) illustrent l'échec de ces tests auprès des type B et $C$, respectivement :

(8) f. * Paul a fait son matériau de prédilection

g. * Paul a fait (avec, à partir de) ce bois son matériau de prédilection

(9) f. * Paul a fait une utilisation intéressante

g. * Paul a fait (avec, à partir de) ce morceau de bois une utilisation intéressante

On peut tirer la conclusion que le complément en de est un complément essentiel dans les phrases des types $\mathrm{B}$ et $\mathrm{C}$, tandis que dans les phrases du type $\mathrm{A}$, il est accessoire et commutable avec d'autres types de compléments prépositionnels.

\subsection{Comportements du $\mathrm{SN}$ direct}


Il existe une série de tests dont l'application à un syntagme nominal directement régi par le verbe s'avère généralement positive et qui constitue donc un ensemble de critères formels pour la reconnaissance de la fonction d'objet direct.

Ces tests divisent les trois types en deux groupes : certains s'appliquent aux trois types, mais d'autres s'appliquent aux types A et C, d'une part, et au type B, de l'autre.

Les tests qui s'appliquent plus ou moins bien aux trois types sont : (a) l'interrogation par qu'est-ce que (ou par que); (b) la relativisation par ce que; (c) la pronominalisation discontinue par en...un d'un $S N$ direct indéfini. Ils s'appliquent bien aux types A et C (cf. $10 \mathrm{a}-\mathrm{c}$ et $12 \mathrm{a}-\mathrm{c}$, respectivement) et moins bien au type B (cf. 11a-c) :

(10) a. Qu'est-ce que Paul a fait de ce type de bois ? - Une belle table

b. Une belle table, c'est ce que Paul a fait de ce type de bois

c. Une belle table, Paul vient d'en faire une de ce type de bois

(11) a. ?Qu'est-ce que Paul a fait de ce type de bois ? - Son matériau de prédilection

b. ?Son matériau de prédilection, c'est ce que Paul a fait de ce type de bois

c. ?Chaque bricoleur a un matériau de prédilection. Paul en a fait un de ce type de bois

(12) a. Qu'est-ce que Paul a fait de ce type de bois ?-C'est une utilisation très intéressante qu'il en a faite

b. Une utilisation intéressante, voilà ce que Paul a fait de ce type de bois

c. On parle souvent à la télé de diverses utilisations intéressantes de matériaux naturels. Paulen a fait une de ce morceau de bois, justement.

Pour ce qui concerne les tests suivants : (d) la pronominalisation par le-la-les; (e) la passivation; et (f) la mise en forme pronominale, les phrases des types $\mathrm{A}$ et $\mathrm{C}$, d'un côté, et les phrases du type B, de l'autre, se comportent d'une façon cohérente. Nous présentons les résultats de l'application avec succès de ces tests aux types $A$ et $C$ en (13d)-(13f) et (14d)(14f), respectivement :

(13) d. Tu vois là-bas une belle table ? C'est Paul qui l'a faite de ce bois

e. Une belle table a été faite de ce bois par Paul

f. Une belle table se fait généralement de ce type de bois

(14) d. L'utilisation qui mérite d'être mentionnée, on l'a faite de ce type de bois, et non de cet autre type

e. Une utilisation intéressante a été faite de ce type de bois par Paul

f. Une utilisation intéressante $s$ 'est faite de ce type de bois par Paul

Voici l'application, en vain, des mêmes tests au type B :

(15) d. * Chaque bricoleur a son matériau de prédilection. Paul l'a fait de ce type de bois

e. *Son matériau de prédilection a été fait de ce type de bois 
f. * Son matériau de prédilection se fait de ce type de bois

\subsection{Double analyse}

L'examen des trois précédentes sections porte sur chacun des trois actants du verbe pris individuellement, dans la relation qu'ils entretiennent avec le verbe. L'application de divers tests examinée ci-dessus indique que les trois types de phrases possèdent la structure syntagmatique suivante :

\section{(16) a. $\quad \mathrm{N} \mathrm{V}[\mathrm{de} N][\mathrm{N}]$}

En plus de l'analyse en trois actants de (16a), l'une des trois constructions examinées possède, cependant, celle en deux actants, auquel cas les deux compléments post-verbaux forment ensemble un syntagme unique. Le schéma suivant illustre une telle analyse de la séquence :

$$
\text { b. } \quad \mathrm{N} V[\mathrm{~N} \text { de } \mathrm{N}]
$$

Le fait qu'une construction syntaxique peut être analysée ou par le schéma du type (16a) ou par celui de (16b) est appelé «double analyse » dans la littérature du lexique-grammaire ${ }^{2}$.

Parmi les trois types que nous examinons, seule le type $\mathrm{C}$ possède la possibilité de double analyse. Une double analyse s'illustre bien avec le test d'extraction par c'est...que, qui donne deux possibilités :

(17) a. C'est une utilisation intéres sante de ce bois que Paul a faite

b. C'est une utilisation intéressante que Paul a faite de ce type de bois

c. C'est de ce type de bois que Paul a fait une utilisation intéressante

Indépendamment de l'analyse en trois actants dans laquelle chacun des deux compléments post-verbaux a une mobilité vis-à-vis du verbe (cf. 17b, c), une phrase du type C possède l'analyse en deux actants, où c'est l'ensemble de deux syntagmes post-verbaux qui forment un syntagme complexe portant une relation vis-à-vis du verbe (cf. 17a).

Les deux autres types ne possèdent pas la double analyse :

(18) a. *C'est une belle table de ce bois que Paul a faite

b. * C'est son matériau de prédilection de ce type de bois que Paul a fait

\subsection{Complément de nom subjectif}

Un nom désignant un «événement » ou un «état » peut construire un syntagme nominal avec ses arguments sous formes de compléments de noms prépositionnels :

(19) a. Le professeur a expliqué le phénomène 

b. l'explication du professeur
c. l'explication du phénomène
d. l'explication du phénomène par le professeur

Autour d'un nom déverbal, par exemple explication, peuvent se construire les noms qui sont interprétables comme ses arguments. En (19b) et (19c), le complément de nom désigne l'argument Agent et l'argument Thème de l'événement désigné par le nom tête explication, respectivement. Cela se voit facilement en se référant à l'exemple (19a) construit avec le verbe expliquer. L'exemple (19d) illustre la réalisation simultanée de ces deux arguments dans un même syntagme au moyen de deux types de compléments de nom différents. L'unique réalisation de l'un des deux arguments se fait au moyen de la préposition de, neutralisant ainsi la différence sémantique de ces deux arguments.

Revenons sur nos premiers exemples. Ce que nous venons de décrire est pertinent pour distinguer un exemple des deux autres. Parmi les trois types, on peut observer une restriction sévère sur l'apparition d'un complément de nom qui reçoit l'interprétation d'agent : c'est le cas du type C. Contrairement aux deux autres, qui acceptent un complément de nom qui a un autre référent que celui du sujet du verbe, dans le cas du type $\mathrm{C}$, cette possibilité n'existe pas :

(20) a. Paul a fait de ce bois la belle table de Luc

b. Paul a fait de ce type de bois le matériau de prédilection de l'école de Vitruve

c. *Paul a fait de ce morceau de bois une utilisation intéressante de Luc

\subsection{Accord}

Un autre trait différencie l'un des trois types. C'est le cas de l'accord entre le $S N$ du complément en de et le $S N$ direct que manifeste seul le type B. Quand c'est possible, cet accord en genre et nombre doit se faire. Voici quelques exemples :

(21) a. Paul voulait faire de ses filles (des avocates, ?*des avocats, *un avocat,

*une avocate)

b. «Plusieurs facteurs se conjuguent pour faire de 2012 une opportunité historique pour faire de la France une championne du numérique... » (lemonde.fr)

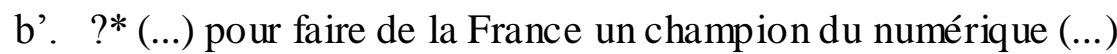

\subsection{Corrélation du déterminant indéfini avec la négation}

Quand la négation dans une phrase "a pour effet de rendre impossible toute véritable quantification", le déterminant indéfini de l'objet direct prend la forme $d e$ :

(22) a. Luc a (un, des) ami(s)

b. Luc n'a pas d'ami(s) ${ }^{4}$ 
Le déterminant indéfini de l'attribut du sujet ne montre pas cette corrélation avec la négation :

$$
\text { Luc n'est pas un ami (cf. * Luc n'est pas d'ami) }
$$

Cette corrélation s'observe avec les types A et C mais pas avec le type B :

(24) a. Paul n'a pas fait de table de ce bois (mais une chaise)

b. Paul n'a pas fait d'utilisation intéressante de ce type de bois

c. * Paul n'a pas fait de ce type de bois de matériau de prédilection

Voici un autre exemple du type B, qui ne vérifie pas la corrélation :

(25) a. «"A ce rythme là, on n'arrivera pas à faire de la France une France de propriétaires", concède le président. » (lemonde.fr)

b. * (...) on n'arrivera pas à faire de la France de France de propriétaires

\subsection{Bilan}

Sept traits ont été pris en compte sur le sujet, le complément en de et le $S N$ direct. De ces observations, on peut tirer des remarques sur chaque construction.

\subsubsection{Support vs. non Support}

Pour ce qui concerne le type A, il est construit sur le verbe faire distributionnel ${ }^{5}$ bi-valent, avec un Agent pour sujet et un Thème (ou Patient) pour objet direct. Le complément en de ne fait pas partie de la valence, il est donc facultatif et substituable par d'autres syntagmes prépositionnels. Le verbe fait partie d'un paradigme constitué de verbes comme créer, fabriquer, qui ont le point commun de prendre un objet effectué ou un objet résultatif.

Le type $\mathrm{C}$ est construit avec le verbe faire, le sujet agentif et l'objet direct. Cette configuration syntaxique est commune au type précédent, mais le type $\mathrm{C}$ diffère du type $\mathrm{Adu}$ fait de la double analyse. Dans la littérature du lexique-grammaire, deux traits auxquels seul le type $\mathrm{C}$ a réagi positivement, c'est-à-dire la possibilité de double analyse (cf. 2.4.) et le refus d'un complément de nom subjectif dont le référent est autre que celui du sujet du verbe (cf. 2.5.) conduisent à différencier fonctionnellement le verbe principal du type $\mathrm{C}$ et celui du type A, bien qu'ils partagent tous les deux la transitivité. Dans le type C, c'est le nom tête du $S N$ direct qui apporte la valence sémantique de la phrase, tandis que le verbe, valenciellement vide, apporte seulement l'information morphosyntaxique nécessaire à son actualisation. Le verbe de ce type est appelé verbe support, différencié du verbe plein ou distributionnel du type A. Le type $\mathrm{C}$ est donc une construction à verbe support ${ }^{6}$.

\subsubsection{Causatif : attribut de l'objet indirect}


La nature fonctionnelle du verbe de la construction du type B est, à son tour, différente de celles des deux autres. En premier lieu, le statut du sujet : contrairement aux deux autres qui nécessitent un sujet agentif selon la valence de l'élément prédicatif (le verbe pour le type A, le nom pour le type C), le sujet de cette construction n'a pas cette restriction (cf. 2.1.). Il est qualifié de «non restreint » dans la classification du lexique-grammaire, acceptant un éventail assez large de formes (complétive, infinitive, nom non humain, etc.). En second lieu, le statut du complément en de : il diffère de celui du type A par le fait de non substituabilité et non omissibilité, et de celui du type $\mathrm{C}$ par le fait de ne pas accepter la double analyse. En dernier lieu, le statut du $S N$ direct : il n'accepte ni le changement de diathèse, ni la corrélation du déterminant indéfini avec la négation. Il montre, en plus, un trait positif que les deux autres ne montrent pas : son accord en genre et en nombre avec le nom du complément en de.

Ces faits indiquent fortement la possibilité d'analyser le $S N$ direct du type B, non comme objet direct, mais comme attribut du complément en de : il s'agit donc d'une construction à attribut (direct) du complément d'objet indirect ${ }^{7}$. Contrairement à la fonction d'attribut de l'objet direct qui a fait l'objet de beaucoup d'études (Nilson-Ehle (1953), Riegel (1974, 1991), Blanche-Benveniste (1991), Muller (2000), Olsson-Jonasson (1981), etc.), dont l'existence est unanimement reconnue, celle d'attribut de l'objet indirect est loin d'être unanimement reconnue. La configuration fonctionnelle que présente le type B est donc insolite.

D'un côté, une grammaire de référence comme Grevisse et Goosse (2011) en mentionne l'existence ${ }^{8}$. Dans la section où est décrit "l'attribut du complément d'objet" (Grevisse et Goosse 2011, §303), on peut lire : “Le complément d'objet est normalement un objet direct. Certains grammairiens considèrent que l'objet peut être indirect."

Après avoir donné des exemples avec des verbes comme se servir et user dans des constructions comme "...en se servant d'un de ses gens comme prêtre et d'un autre comme témoins (S.-Beuve)" ou "Il use de cette plante comme remède", dans lesquels il s'agit non seulement d'un attribut d'objet indirect mais aussi d'un attribut indirect, cette grammaire donne, justement, un exemple de l'attribut du complément d'objet indirect avec le verbe faire, qui correspond à notre type B : "Milady laissa tomber sur lui un de ces regards qui d'un esclave font un roi (A. Dumas)."

Par ailleurs, certains linguistes nient explicitement l'existence de cette fonction grammaticale. C'est le cas notamment de Herslund (1988a, 1988b, 1994, 2004), Herslund \& Sørensen (1994), qui ont proposé une théorie syntaxique basée sur la théorie de la valence : selon eux, la valence verbale est constituée d'un argument fondamental (le sujet de l'intransitif ou l'objet direct du transitif), et d'un sujet (dans le cas du transitif). Le verbe et son argument fondamental constitue un fondement prédicatif. Le prédicat ainsi constitué et le sujet forment une prédication, pour former ensemble une proposition. D'autres compléments verbaux non circonstanciels, comme un complément datif, locatif, etc., ou un attribut (du sujet ou de l'objet direct) assument une fonction grammaticale qu'ils ont appelé Adjet ${ }^{9}$, dont la particularité est qu'il entretient à la fois une relation avec le verbe et une relation avec un des actants du verbe (le sujet ou l'objet direct). Cette dernière est une relation de prédication seconde. De ce point de vue, on peut en effet exclure une fonction grammaticale comme attribut de l'objet indirect, puisque ce serait un cumul de deux Adjets, ce qui est a priori exclu de la typologie des phrases en français. 
Malgré son statut instable, nous avançons que la fonction d'attribut de l'objet indirect existe $^{10}$ et que le $S N$ direct du type B a une telle fonction. Cette hypothèse est jusqu'ici appuyée par les oppositions syntaxiques que le $S N$ direct manifeste par rapport au véritable objet direct qu'on peut observer dans les deux autres constructions du même verbe faire. Dans la section suivante, nous présentons d'autres arguments qui appuieraient cette hypothèse : il s'agira de la position qu'occupe la construction en question dans la distribution des formes causatives des phrases copulatives.

En effet, le caractère non restreint du sujet de la construction du type B permet de la comparer à un autre type de construction du verbe faire : il s'agit d'une construction factitive. La phrase du type B (26a) peut être paraphrasée par une phrase factitive $(26 b)^{11}$ :

(26) a. (Son père, sa détermination) a fait de Paul un chercheur en mathématiques

b. (Son père, sa détermination) a fait que Paul est un chercheur en mathématiques

En guise de résumé de la discussion ci-dessus, Tableau 1 récapitule des traits syntaxiques et sémantiques de chaque constituant, ainsi que des traits qui concernent plus d'un constituant, dans chacune des constructions.

\begin{tabular}{|c|c|c|c|c|c|c|c|c|c|c|c|c|c|c|c|c|c|c|}
\hline & $\mathrm{S}$ & \multicolumn{7}{|c|}{ Oblique } & \multicolumn{6}{|c|}{$\mathrm{O}$} & \multirow[b]{2}{*}{$\begin{array}{l}\bar{z} \\
0 \\
\bar{z} \\
z \\
z \\
z\end{array}$} & \multirow[b]{2}{*}{$\begin{array}{l}> \\
\bar{z}\end{array}$} & \multirow[b]{2}{*}{ 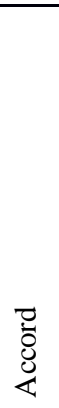 } & \\
\hline & 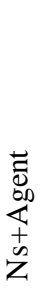 & $\begin{array}{l}\tilde{0} \\
\text { II } \\
z \\
\text { I }\end{array}$ & $\begin{array}{l}\tilde{\theta} \\
\overrightarrow{0} \\
\bar{y} \\
\| 1 \\
z \\
\overline{0}\end{array}$ & $\begin{array}{l}\vec{z} \\
\text { I } \\
\text { II } \\
z \\
\text { z }\end{array}$ & 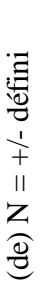 & 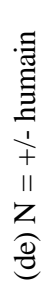 & $\begin{array}{l}0 \\
\text { II } \\
z \\
0\end{array}$ & 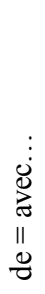 & 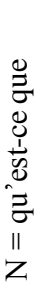 & $\begin{array}{l}\circlearrowright \\
\Xi \\
\Xi \\
I I \\
z\end{array}$ & 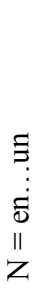 & 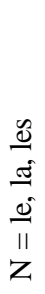 & $\stackrel{4}{n}$ & 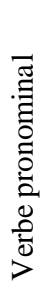 & & & & 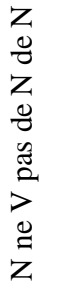 \\
\hline (1) & + & + & + & + & + & + & + & + & + & + & + & + & + & + & - & + & - & + \\
\hline (2) & \pm & + & + & + & + & + & - & - & + & + & + & - & - & - & -1 & + & + & - \\
\hline (3) & + & + & + & + & + & + & - & - & + & + & + & + & + & + & + & - & 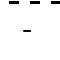 & + \\
\hline
\end{tabular}

Tableau 1 : Traits syntaxiques et sémantiques des constructions

Les types A-B-C peuvent être analysés en (27a)-(27c), respectivement :

(27) a. (N+Agent)Sujet Vdistributionnel (de, a vec N)Obl. (N)Objet

b. (N土Agent)Sujet Vcausatif (de N)Obj_ind. (N)Attribut

c. $\quad(\mathrm{N}+$ Agent)Sujet Vsupport (de N)Obj_ind. (N)Objet

$=(\mathrm{N}+$ Agent $)$ Sujet Vsupport $(\mathrm{N}$ de $\mathrm{N})$ Objet

Dans ce qui suit, les types A et $\mathrm{C}$ seront laissés de côté, au profit du type B, qui sera analysé comme une construction avec l'emploi causatif attributif du verbe faire. 


\section{Faire verbe causatif s'appliqua nt à des phrases copulatives bi-nominales}

Dans la section précédente, trois types de constructions syntaxiques du verbe faire qui présentent la même forme de surface ont été comparés et un type s'est avéré digne d'intérêt spécial parce que le $S N$ direct n'y présente pas de caractères de l'objet direct, contrairement aux deux autres. Des faits de l'accord et de son refus de la promotion en sujet, ce constituant a été qualifié d'attribut. Il est en effet possible de constater l'existence d'une relation de prédication entre le complément en de et le $S N$ direct dans la construction en question.

Dans ce qui suit, il sera présenté de nouveaux arguments basés sur deux autres comparaisons pour renforcer cette analyse : d'abord, la relation prédicative entre ces deux compléments sera mieux caractérisée, au regard de la typologie des phrases copulatives; ensuite, il sera réfléchi au paradigme de formes causatives des phrases copulatives.

En synthèse, il sera montré que la construction en question est une forme causative particulière d'un type de phrase copulative. Ces considérations permettent de combler un trou de distribution dans le ledit paradigme et d'aligner le verbe faire sur d'autres verbes causatifs dont le statut est traditionnellement reconnu, comme rendre et mettre.

\subsection{Typologie des phrases copulatives}

Il est bien connu que la configuration syntaxique simple Sujet-être-Attribut que présentent les phrases copulatives peut être subdivisée en plusieurs sous-types, en fonction des différences formelles et interprétatives des deux éléments constitutifs de la phrase. Nous présentons deux typologies des phrases copulatives bien connues et examinons si la relation entre (de) $N$ et $N$ du type B peut être identifiée au moyen de ces typologies.

\subsubsection{Riegel (1985)}

Riegel (1985) distingue quatre types d'attributs du sujet, selon la catégorie grammaticale de l'élément qui l'assume et selon le type de détermination, dans le cas où cet élément est nominal :

(28) a. $\quad \mathrm{N}$ être ADJ $=$ : Luc est gentil

b. $\quad \mathrm{N}$ être UN $\mathrm{N}=$ : Ce meuble est un vaisselier

c. $\quad \mathrm{N}$ être $\mathrm{LEN}=$ : Mon voisin est le champion du monde d'haltérophilie

d. $\quad \mathrm{N}$ être $\mathrm{N}=$ : Luc est professeur

Dans la phrase (28a) et dans l'une des deux interprétations possibles de la phrase (28d), l'attribut du sujet, réalisé par un adjectif et un syntagme nominal sans déterminant respectivement, a une fonction qualifiante. Ce type d'attribut répond à la question en comment. Dans les phrases (28b-c), et dans l'autre interprétation possible de la phrase (28d), l'attribut du sujet, réalisé par des syntagmes nominaux indéfini, défini et sans déterminant respectivement, joue le rôle typant, répondant à la question en que.

\subsubsection{Van Peteghem (1991)}


Van Peteghem (1991) est une étude sur les phrases copulatives de la forme $S N$ être $S N$ basées sur la typologie de Higgins (1973) ${ }^{12}$. Selon cette étude, les phrases copulatives dont l'attribut du sujet est un syntagme nominal peuvent se répartir en trois classes :

(29) a. Jean Dupont est professeur de latin

b. Cet homme est un professeur de latin

c. Son professeur de latin est Jean Dupont

La phrase (29a) représente le type prédicationnel, avec une structure informative non marquée. La phrase (29b) est une phrase identificationnelle, avec un sujet qui contient une information connue et un attribut qui fournit une information nouvelle. La phrase (29c) est une phrase spécificationnelle, dans laquelle le sujet de surface est considéré comme un attribut antéposé (ou attribut profond) assumé généralement par un syntagme nominal défini qui implique la présupposition d'existence, et l'attribut de surface est un sujet postposé (ou sujet profond). Les traits d'interprétation du type spécificationnel de phrase copulative se résument aux deux points : (a) le sujet sert d'étiquette qui caractérise un ensemble, décrivant de la sorte les conditions que doivent satisfaire tous les éléments de cet ensemble et (b) l'attribut donne une valeur particulière qui satisfait ces conditions. Van Peteghem (1991) n'attribue pas un type particulier à une phrase dans laquelle l'ordre des constituants d'une phrase spécificationnelle est inversé, c'est-à-dire une phrase normale avec l'ordre de Sujetcopule-Attribut. Par définition, elle n'est plus spécificationnelle.

Si on compare la classification de Riegel (1985) et celle de Van Peteghem (1991), on peut établir le Tableau 2 :

\begin{tabular}{|c|c|c|}
\hline & Riegel (1985) & Van Peteghem (1991) \\
\hline $\mathrm{N}$ être ADJ (25a) & \multirow{2}{*}{ « qualifiant » } & (non traité) \\
\hline \multirow{2}{*}{ N être N (25d), (26a) } & & «prédicationnel $»$ \\
\hline & \multirow{3}{*}{ «typant » } & - \\
\hline N être UN N (25b), (26b) & & « identificationnel » \\
\hline N être LE N (25c) & & - \\
\hline LE N être N (26c) & & « spécificationnel» \\
\hline
\end{tabular}

Tableau 2 : Comparaison entre Riegel (1985) et Van Peteghem (1991)

\subsection{Types de relation entre deux $\mathrm{SN}$}

On a présenté l'hypothèse que le $S N$ direct de la construction du type B fonctionne comme attribut du $S N$ qui se trouve sous la préposition $d e$. Il sera vérifié dans ce qui suit si les mêmes types de relation reconnus entre le sujet et son attribut dans les phrases copulatives se trouvent également entre ces deux termes, sur la base de la classification de Van Peteghem (1991) présentée ci-dessus .

\subsubsection{Type prédicationnel exclu}


Dans la position du $S N$ direct, on ne peut trouver ni l'adjectif ni le nom sans déterminant, deux catégories représentatives de l'interprétation prédicationnelle de l'attribut. Il en résulte que dans la construction iciétud iée, l'interprétation prédicative est exclue :

(30) a. *On a fait de Jean (intelligent, beau, timide, etc.)

b. * On a fait de Jean (très professeur, très médecin, très français, etc.)

c. *Comment a-t-on fait de Jean?

Les exemples (30a) montrent l'impossibilité pour un adjectif d'apparaître dans la position du $S N$ direct, ceux de (30b) l'impossibilité d'y trouver un nom sans déterminant (le modifieur très est ajouté pour souligner l'interprétation prédicationnelle d'un tel constituant). Il va de soi, sous cette condition, qu'il est exclu d'interroger le complément direct par comment (cf. 30c), ce qui est un caractère formel de l'attribut prédicationnel.

\subsubsection{Type identificationnel accepté}

Le rapport entre les deux compléments est comparable à celui qu'on trouve dans une phrase copulative identificationnelle dans les phrases suivantes :

(31) a. (Son père, son éducation) a fait de Luc un professeur de latin

b. «Il avait séduit G. Pompidou qui, dit T. Desjardins, en (=C. Pasqua) aurait fait un ministre après mai 68 , s'il était lui-même resté à la tête du gouvernement. » (Le Monde, 1994)

c. La nouvelle classification internationale des meubles a fait de ce meuble un vaisselier

Dans les exemples (31a-c), on peut reconnaître les phrases copulatives suivantes, qui sont analysables comme identificationnelles :

(31) a'. Luc est un professeur de latin

b'. C. Pasqua est un ministre

c'. Ce meuble est un vaisselier

\subsubsection{Type spécificationnel exclu}

Le $S N$ régi par la préposition de et le $S N$ direct ne semblent pas pouvoir entretenir une prédication spécificationnelle :

(32) a. * La police a fait de l'homme qui a tué Ida Jean Dupont

(cf. L'homme qui a tué Ida est Jean Dupont)

b. La police a fait de Jean Dupont l'homme qui a tué Ida

(cf. Jean Dupont est l'homme qui a tué Ida)

(33) a. «(..) il a inventé un art sans devancier ni, pour l'instant, imitateur, qui a fait de lui le maître du bambou »(Le Monde, 1994)

b. * (...), qui a fait du « maître du bambou » cet homme 
(34) a. «M. Galeazzi a fait de J. Chouraqui et d'A. Gallo les commanditaires du meurtre de L. Mout » (Le Monde, 1994)

b. * M. G. a fait des commanditaires du meurtre de L. Mout J. Chouraqui et A. Gallo

L'exemple (32a) montre l'impossibilité de trouver le sujet d'une phrase spécificationnelle comme complément en de et son attribut comme $S N$ direct $^{13}$. Si on inverse l'ordre de ces deux constituants, la phrase est acceptable (32b).

Les couples de phrases (33) et (34) montrent qu'à partir des exemples acceptables (33a) et (34a) où c'est la relation identificationnelle qui est représentée par les deux compléments du verbe faire, il n'est pas possible de construire des phrases où l'ordre des deux éléments est inversé, en sorte que ce soit la relation spécificationnelle qui s'y trouve (cf. 33b, 34b).

\subsubsection{Bilan}

La comparaison ci-dessus donne une conclusion assez simple : la seule relation prédicative qu'on peut reconnaître entre les deux compléments $\mathrm{du}$ verbe faire est celle appelée identificationnelle dans la typologie des phrases copulatives, au détriment des prédicationnelle et spécificationnelle, sur la base de la terminologie de Van Peteghem (1991).

La raison pour laquelle le type prédicationnel est exclu avec le verbe faire est formelle : l'adjectif et le nom sans déterminant sont exclus de la position du $S N$ direct dans cette construction ${ }^{14}$.

Pour quelle raison le type spécificationnel est-il écarté de cette construction? Ce fait de distribution qui paraît arbitraire est, en fait, conforme à notre hypothèse selon laquelle le $S N$ sous de correspond au sujet, et le $S N$ direct à l'attribut. Or, selon Van Peteghem (1991), le sujet (ou le $S N$ pré-copule) d'une phrase spécificationnelle est un attribut antéposé (ou profond) et son attribut (ou le $S N$ post-copule) un sujet postposé. Il est donc naturel qu'une phrase spécificationnelle ne puisse pas être intégrée en maintenant l'ordre des constituants dans une construction avec faire. L'ordre de (de) SN SN correspond en quelque sorte à la configuration canonique des fonctions grammaticales, Sujet-Attribut. Ainsi, les données observées servent à conforter, à la fois, l'analyse de la phrase spécificationnelle de Van Peteghem (1991) et notre hypothèse.

\subsection{Phrases causatives des phrases copulatives}

Dans le cadre de la grammaire transformationnelle inspirée de Z. Harris, Gross (1981) a proposé d'analyser les verbes mettre et rendre comme des opérateurs causatifs, qui s'appliquent à des phrases de base copulatives, en augmentant de un le nombre d'actants d'une phrase résultante et en y apportant le sens causatif. Imaginons des phrases copulatives de départ :

$$
\begin{aligned}
& \text { a. } \quad \mathrm{N} \text { être } \mathrm{ADJ}=\text { : L' eau est rouge } \\
& \text { b. } \quad \mathrm{N} \text { être Prép } \mathrm{N}=\text { : Luc est en rage }
\end{aligned}
$$


Elles peuvent avoir des phrases causatives correspondantes, qu'on peut décomposer de la manière suivante :

(36) a. $\quad \mathrm{N}$ rendre \# $\mathrm{N}$ être $\mathrm{ADJ}=$ : (Le sang, Paul) a rendu l'eau rouge

b. $\quad \mathrm{N}$ mettre \# $\mathrm{N}$ être Prép $\mathrm{N}=$ : (La désinvolture de Léa, Paul) a mis Luc en rage

Les phrases copulatives de (35) sont toutes les deux du type prédicationnel. Les phrases de (36) sont, en revanche, des phrases causatives dans lesquelles les phrases de (35) se trouvent enchâssées, suite à l'application de la séquence causative de la forme $N$ Vcausatif qu'on représente par le signe \#. Les verbes rendre et mettre sont en distribution complémentaire selon la catégorie grammaticale qui assume la fonction d'attribut prédicationnel ${ }^{15}$ : s'il est adjectival, le causativation se fait avec le verbe rendre, et s'il est assumé par un syntagme prépositionnel adjectival, elle se fait avec le verbe mettre.

L'intérêt de reconnaître le statut d'opérateur causatif à ces deux verbes est qu'il remédie lexicalement à l'absence bien connue de la construction causative infinitivale des phrases copulatives. En effet, comme l'a déjà remarqué Kayne (1977 : 239), le verbe causatif faire ne peut pas en général se construire avec l'infinitif être :

* Cela a fait être son fils malade

L'application du causatif infinitival faire aux phrases copulatives de (35) donne ainsi des résultats inacceptables :

a. *(Le sang, Paul) a fait être rouge (à/par) l'eau

b. * (La désinvolture de Léa, Paul) a fait être en rage (à/par) Luc

Le manque syntaxique de la distribution du verbe causatif infinitival faire dans des environnements comme (37) et (38) est lexicalement contourné au moyen de verbes intrinsèquement causatifs comme rendre et mettre : les phrases du type (36) suppléent en effet à la distribution manquante du type (38). Les constructions syntaxiques des verbes rendre et mettre sont des versions causatives de phrases copulatives prédicationnelles dans lesquelles la relation prédicative Sujet-Attribut d'une phrase copulative est réalisée comme celle entre Objet Direct et Attribut d'Objet Direct.

Il a été observé que la séquence des compléments (de) $N N$ de la construction $N$ faire de $N N$ ne peut pas entretenir une relation prédicationnelle. Du point de vue de la distribution des phrases causatives et des phrases copulatives, ce trou de distribution n'a rien de gênant : il s'avère qu'il est simplement comblé par des constructions avec les verbes rendre et mettre. Par ailleurs, il est intéressant de noter que ces deux derniers n'acceptent pas d'attribut nominal: on peut en déduire qu'ils ne peuvent s'appliquer ni à une copulative identificationnelle, ni à une copulative spécificationnelle, constituant ainsi un autre trou de distribution. 
A l'inverse, il est possible de considérer que c'est précisément la construction $N$ faire de $N N$, cette fois, qui supplée à l'un des deux trous, au moins : elle constitue une phrase causative des phrases copulatives identificationnelles. Dans la littérature, il ne semble pas que ce fait, c'est-à-dire le constat de cases vides dans la distribution des phrases causatives des phrases copulatives, ait jamais été remarqué. L'hypothèse de l'attribut du complément indirect de la construction avec faire et la mise en parallèle de celle-ci avec des phrases avec rendre et mettre ont permit de découvrir ces cases vides. Elles sont désormais à moitié remplies.

\subsection{Distribution des phrases causatives des copulatives}

La distribution des phrases causatives des copulatives est résumée dans le tableau 3.

\begin{tabular}{|c|c|c|}
\hline Copulatives & Types & Causatif \\
\hline $\mathrm{N}$ être ADJ & \multirow{3}{*}{ « qualifiant/prédicationnel» } & $\mathrm{N}$ rendre $\mathrm{N}$ ADJ (cf. 33a) \\
\hline N être Prép N & & $\mathrm{N}$ mettre N Prép N (cf. 33b) \\
\hline \multirow{2}{*}{$\mathrm{N}$ être $\mathrm{N}$} & & $\mathbf{X}$ \\
\hline & «typant » & $\mathbf{Y}$ \\
\hline $\mathrm{N}$ être UN N & «typant/identificationnel» & $\mathrm{N}$ faire de $\mathrm{N} \mathrm{UN} \mathrm{N}$ (cf. 31a) \\
\hline $\mathrm{N}$ être LE N & «typant » & $\mathrm{N}$ faire de $\mathrm{N}$ LE N (cf. 32b) \\
\hline LE N être $\mathrm{N}$ & «spécificationnel» & $\mathbf{Z}$ \\
\hline
\end{tabular}

Tabelau 3 : Distribution des formes causatives

Dans la distribution des formes causatives, il reste trois cases vides, que nous avons appelées $\mathrm{X}, \mathrm{Y}$ et $\mathrm{Z}$. Les causatives qui remplissent les cases $\mathrm{X}$ et $\mathrm{Y}$ correspondent à celles dans lesquelles se trouvent deux interprétations différentes de l'attribut qui se réalise par un nom sans déterminant, prédicationnel et identificationnel, respectivement. La case $\mathrm{Z}$ concerne la causativation d'une phrase spécificationnelle. Nous examinons dans ce qui suit quelques hypothèses pour chaque cas de figure.

\subsubsection{Case X}

La phrase copulative de départ est la suivante :

Luc est (très prof, encore très étudiant, très enfant)

L'attribut du sujet est assumé par un nom sans déterminant avec la fonction adjectivale, ce qui est souligné par le modifieur adverbial de degré très. Dans cette fonction, selon Riegel (1985 : 195), le nom sans déterminant "a la particularité de dénoter une propriété ou un complexe de propriétés pragmatiquement interprétés comme caractéristiques des individus de la classe N1". Selon le même auteur, dans le paradigme de la copule être se trouvent également des locutions comme donner l'impression d'être un / avoir l'apparence extérieur d'un / avoir le comportement d'un, etc ${ }^{16}$. L'attribut de ce type a la particularité de répondre à 
la question en comment, comme une copulative adjectivale et se distingue ainsi de l'emploi identificationnel de la même forme qui répond à la question en que.

Les constructions du type (39) ne sont pas mises en formes causatives par les verbes rendre et mettre :

$$
?^{*}(\text { Cet habit, son père) a (rendu, mis) Luc très (prof, enfant, étudiant, etc.) }
$$

Avec le verbe faire, il est exclu de construire ces noms à la place du $S N$ direct :

$$
\text { *(Cet habit, son père) a fait de Luc très (prof, enfant, étudiant, etc.) }
$$

Mais dans une autre construction du même verbe, ces mêmes noms semblent se construire comme attribut :

$$
\text { (Cet habit, ça) fait très (prof, enfant, étudiant, etc.) }
$$

Or, la difficulté de considérer les phrases (42) comme des formes causatives de l'exemple (39) émane du fait qu'il est très difficile de construire l'objet sur lequel porte les attributs (c'est-à-dire le sujet des phrases (39)). Lauwers (2008) mentionne lui aussi la possibilité d'analyser ce type de phrases comme phrases causatives, mais il a opté pour les analyser comme des phrases copulatives avec sujet métonymique.

A ce stade de travail, il faut conclure qu'on ne trouve pas de version causative lexicalisée de ce type de construction copulative. La causative infinitivale peut être construite sur sembler ou paraître :

$$
\text { (Cet habit, ça) fait (sembler, paraître) Luc très (prof, enfant, étudiant, etc.) }
$$

Mais elle ne peut jamais être construite ni avec le verbe être ni avec le verbe faire :

$$
\text { *(Cet habit, ça) fait (être/faire) Luc très (prof, enfant, étudiant, etc.) }
$$

\subsubsection{Case $Y$}

Le matériel lexical qui constitue l'attribut est le même que celui du cas précédent, mais il y a une différence entre le type de construction examiné ici et celui examiné dans la section précédente : dans l'interprétation pertinente, la phrase $N$ être $N$ implique nécessairement $N$ être $U N N$;

(45) a. Luc est (professeur, étudiant)

b. Luc est très (prof, étudiant)

c. Luc est un (professeur, étudiant)

Les exemples (45a) impliquent nécessairement les exemples (45c), tandis que les exemples (45b) que nous avons examinés dans la section précédente ne les impliquent pas. 
Une autre différence est que l'attribut de cette construction répond à la question en quelqui et non en comment.

Aucune des constructions causatives étudiées ici n'acceptent l'attribut de l'interprétation de (45a) :

(46) a. *Ses parents ont (rendu, mis) Luc (professeur, étudiant)

b. *Ses parents ont fait de Luc (professeur, étudiant)

Avec faire, cependant, selon les noms qui assument la fonction de l'attribut, une construction à attribut de l'objet direct est possible :

(47) a. «L'air tendu, F. Hollande est fait Grand maître de la Légion d'honneur, en présence des présidents du Sénat, J.-P. Bel et de l'assemblée, l’UMP B. Accoyer. » (publicsenat.fr)

b. F. Hollande est Grand maître de la Légion d'honneur.

c. «N. Moretti a été fait commandeur des arts et des lettres. »(parimatch.com)

d. N. Moretti est commandeur des arts et des lettres.

Dans ces constructions, le verbe faire a exactement la même valeur que le verbe nommer et comme c'est le cas avec ce dernier, il impose la restriction de sélection sur le sujet, l'objet direct et son attribut. Ce dernier doit être un nom qui désigne une fonction ou un statut officiels auxquels on accède par le biais d'un procès de nomination socialement codifié. Il en résulte que tous les noms sans déterminant qui peuvent fonctionner comme attribut du sujet ne peuvent pas se trouver comme attribut de l'objet direct dans ces constructions causatives :

a. ?*Luc a été (fait, nommé) (étudiant, lycéen, collégien, etc.)

b. Luc est (étudiant, lycéen, collégien, etc.)

Ainsi, pour qu'on puisse dire qu'il s'agit d'opérateurs causatifs, les verbes nommer et faire manquent de généralité. Tout au plus, ce sont des opérateurs qui s'appliquent à un soustype des copulatives identificationnelles avec un nom sans déterminant.

\subsubsection{Case Z}

Voici des exemples de phrases spécificationnelles :

(49) a. Son professeur de latin est Jean Dupont

b. Le champion du monde d'haltérophilie est mon voisin

Nous avons vu en 3.2.3. que la construction faire de $N N$ ne peut pas constituer une variante causative des phrases du type (49). Il va sans dire que les verbes rendre et mettre ne conviennent pas non plus. Peut-on imaginer une variante causative de ces phrases? 
3.4.3.1. $N$ avoir pour $N N$. Des phrases comme les suivantes semblent comporter la relation spécificationnelle qu'on observe dans les exemples (49) entre le $N$ régi par la préposition pour et le $S N$ direct :

(50) a. Il a pour professeur de latin Jean Dupont

b. Le monde a pour champion d'haltérophilie mon voisin

Examinons quelques exemples des constructions similaires :

(51) a. «Le supplicié du soir a pour nom R. M., le député UMP sortant, que la ministre (...) a défait dans la 5è circonscription des B.-R., avec un peu plus de 1.400 voix d'a vance. » (lemonde.fr)

b. «Pour l'organisateur et créateur du concours, F. R., journaliste et animateur, le titre "Miss Black France" a pour objectif de "célébrer la beauté noire", (...)» (lemonde.fr)

c. «J'affirme haut et fort que l'électricité que l'Allemagne nous a vendue avait pour origine leurs centrales au gaz et au charbon. » (lemonde.fr)

Les phrases (51) ont toutes la construction schématisée en (52a). Dans chacune de ces phrases, la relation entre le $N$ sans déterminant régi par la préposition pour et le $S N$ direct du verbe avoir peut être exprimée par une phrase copulative spécificationnelle, que nous donnons en (52b-d), qu'on peut schématiser par (52e) :
a. $\quad \mathrm{Nb}$ avoir pour $\mathrm{Na} \mathrm{Nc}$
b. Le nom du supplicié du soir est R. M.
c. L'objectif du titre «Miss Black France » est de «célébrer la beauté noire »
d. L'origine de l'électricité que l'Allemagne nous a vendue était leurs centrales au gaz et au charbon
e. $\quad \mathrm{Na}$ de $\mathrm{Nb}$ est $\mathrm{Nc}$

Il est impossible de considérer, pourtant, les propositions de la forme (52a) comme des variantes causatives des copulatives spécificationnelles, pour une raison précise : dans les premières, il n'est pas exact de dire que le nombre d'arguments s'est augmenté de un par rapport à ces dernières. Or, c'est là la définition de l'application d'un opérateur causatif. En effet, la relation entre ces deux types de phrases est plutôt celle de reformulation, de façon que le sujet du verbe avoir $(\mathrm{Nb})$ est, dans les cas les plus faciles à observer, le possesseur du nom tête du sujet de la phrase copulative $(\mathrm{Na})$, et même dans les cas moins clairs, celui qui s'exprime sous forme d'adjectif possessif ou de complément génitif par rapport à ce nom tête.

La relation de possession est ici comparable à la relation de possession inaliénable que l'on observe dans une construction à attribut de l'objet direct du verbe avoir (51a, cf. Furukawa (1987), Riegel (1988, 1998), Kupferman (2000)), qu'on peut paraphraser elle aussi par une phrase copulative (53b) : 
(53) a. $\quad \mathrm{Nb}$ avoir $\mathrm{Na} \mathrm{Adj}=:$ Luc a les yeux bleus

b. $\quad \mathrm{Na}$ de $\mathrm{Nb}$ est $\mathrm{Adj}=$ : Les yeux de Luc sont bleus

La relation d'équivalence entre (53a-b) est similaire à celle qu'on constate entre (52a) et (52e). Les différences entre la phrase du type (51a) et celle du type (52a) sont les faits suivants : (a) le sujet de la phrase copulative se réalise comme objet direct défini en (53a) et comme complément sous la préposition pour en (52a); (b) la relation de possession inaliénable est marquée par le déterminant défini en (53a), tandis qu'elle semble se réaliser par la cooccurrence de la préposition pour et l'absence du déterminant en (52a); (c) l'attribut de l'objet direct est assumé par un adjectif ou ses équivalents (syntagmes prépositionnels) en (53a), tandis que dans le cas de l'exemple du type (52a), l'attribut du complément en pour est exclusivement assumé par un syntagme nominal.

Les phrases (50) ne sont certes pas des phrases causatives ordinaires par rapport aux phrases (49), mais la relation entre le $N$ sans déterminant de pour $N$ et le $S N$ direct de avoir est tout à fait comparable à la relation sujet et attribut d'une phrase copulative spécificationnelle : si on s'en tient aux relations grammaticales de surface, la construction $N$ avoir pour $N N$ présente un autre cas de figure où le $S N$ direct sert d'attribut à un syntagme nominal introduit par une préposition (i.e., attribut de l'objet ind irect).

Cependant, au regard de l'hypothèse selon laquelle une phrase spécificationnelle est une phrase inversée où l'élément pré-copule est mieux analysé comme un attribut profond et l'élément post-copule comme un sujet profond, le véritable sujet dans la construction avec avoir est le $S N$ direct ( $N c$ de $52 \mathrm{a}$ ) et le véritable attribut le complément indirect ( $N a$ de $52 \mathrm{a}$ ). Donc c'est une construction à attribut indirect de l'objet direct ${ }^{17}$.

3.4.3.2. Verbes à interrogatives indirectes partielles. Une des caractéristiques d'une copulative spécificationnelle est qu'elle accepte une question en quel :
a. Quel est le nom du supplicié du soir ?
b. Quel est l'objectif du titre « Miss Black France »?
c. Quelle est l'origine de l'électricité que l'Allemagne nous a vendue ?

Par ailleurs, selon notamment les études de Muller $(1989,1996)$ et de Nakamura (2008) à sa suite, toute proposition interrogative indirecte partielle (IIP) est réductible à la forme équivalente quel est $L E N$ où le $N$ est occupé par un nom classifieur approprié à l'élément $Q u$ propre à chaque proposition interrogative. Par exemple, voici une paire de paraphrases pertinente à ce propos :

(55) a. Je sais qui vient d'entrer

b. = Je sais quelle est la personne qui vient d'entrer (Muller 1996 : 198)

L'IIP qui vient d'entrer de (55a) est substituable par une IIP équivalente quelle est la personne qui vient d'entrer de (55b). Le nom approprié personne dans la seconde proposition 
est choisi en fonction de l'élément $Q u-=q u i$ de la première. En totalité, cette substitution maintient l'équivalence interprétative des deux phrases de (55).

Or, selon Van Peteghem (1991 : 72-74), entre autres, l'interrogatif quel est spécialisé pour identifier l'élément post-copule ${ }^{18}$ d'une copulative spécificationnelle (et il peut très rarement remplacer l'attribut d'une identificationnelle).

Par ailleurs, certains verbes introducteurs d'une IIP acceptent la réduction de quel est, pour ne laisser à la surface que le $S N=$ le $N$ (Modif) et la phrase résultante maintient toujours l'interprétation d'IIP. C'est le phénomène dit de la question cachée :

(56) a. Robert a (demandé, précisé, indiqué...) où sa femme avait laissé sa voiture

b. = Robert a (demandé, préc isé, indiqué...) quelle était l'endroit où sa femme avait laissé sa voiture

c. = Robert a (demandé, précisé, indiqué...) l'endroit où sa femme avait laissé sa voiture

d. L'endroit où sa femme avait laissé sa voiture est $\mathrm{X}$

De ces observations, on peut former l'hypothèse que les verbes à IIP pourraient être des verbes causatifs qui s'appliquent à une phrase copulative spécificationnelle, mais non dans sa totalité : l'assertion des phrases comme (56b-c) signifie, en effet, qu'un actant sujet indépendant de la phrase copulative (cf. 56d) effectue un procès abstrait (de demande, précision ou indication) dont l'aboutissement implique l'assertion de la phrase copulative spécificationnelle, mais le contenu de la prédication n'est pas présenté dans ces cadres de phrase.

Il semble qu'on peut identifier une contrepartie non interrogative et explicite où la prédication copulative impliquée dans le procès est clairement explicitée :

(57) a. Robert a (*demandé, précisé, indiqué) l'endroit où sa femme avait laissé sa voiture comme étant le parking devant la gare

b. L'endroit où sa femme avait laissé sa voiture est le parking devant la gare

Par rapport à la phrase copulative spécificationnelle (57b), les phrases (57a) pourraient être considérées comme une version causative où un actant supplémentaire par rapport à la phrase de base apparaît comme sujet : il effectue un procès de précision ou d'indication dont l'issue est l'assertion de la phrase spécificationnelle (57b). La partie attributive est introduite par une proposition participiale, régie par la conjonction comme. La suppression de étant semble difficile :

?* Robert a (précisé, indiqué) l'endroit où sa femme avait laissé sa voiture comme (le) parking devant la gare

Il est cependant intéressant de noter que la possibilité de la suppression de étant dépend, à la fois, du verbe principal et de l'ordre des $S N$ (cf. 59a) : 
(59) a. Robert a (?précisé, indiqué) le parking devant la gare comme (l')endroit où sa femme avait laissé sa voiture

b. Robert a (précisé, indiqué) que le parking devant la gare était l'endroit où sa femme avait laissé sa voiture

La comparaison des exemples de (59a) avec ceux de (59b) suggère la possibilité de considérer la structure $N$ comme $N$ des premiers comme une réduction de la complétive copulative des derniers. Dans ce cas-là, le complément en comme est un attribut indirect de l'objet direct qui est le sujet d'une copulative identificationnelle de la complétive. La relation n'est plus spécificationnelle.

\subsubsection{Bilan}

La distribution des formes causatives dépend des types de phrases copulatives : il a été constaté ci-dessus l'existence de « trous » de distribution illustrée dans le Tableau 3 et il a été examiné s'il est possible de combler ces trois cases vides au moyen du verbe faire, ou d'autres verbes, le cas échéant.

Pour ce qui concerne la case $\mathrm{X}$, correspondant à la forme causative d'une phrase copulative prédicationnelle dont l'attribut se réalise au moyen d'un nom sans déterminant, il n'est pas possible de trouver une construction causative attributive qui intègre une seconde prédication. La forme causative de cette classe est assumée par une causative infinitivale dont l'infinitif est réalisé par des verbes comme paraître ou apparaître, avec lesquels la copule être de base est en alternance libre dans la construction copulative même.

Pour ce qui concerne la case Y, qui correspond à la forme causative d'une construction copulative identificationelle dont l'attribut se réalise par un nom sans déterminant, une construction à attribut de l'objet direct du verbe faire semble, à première vue, la combler. Or, dans cette construction, le verbe impose une certaine restriction de sélection, comme les verbes élire et nommer qui se trouvent dans le même paradigme : le sujet doit être interprété comme agent. On peut considérer donc qu'il s'agit d'un faire causatif lexical, et non d'un faire opérateur causatif.

Pour ce qui concerne la case $\mathrm{Z}$, qui correspond à la forme causative d'une construction copulative spécificationnelle, la première hypothèse explorée est de voir en la construction $N$ avoir pour $N N$ une sorte de variante causative de la phrase spécificationnelle. Or, ce n'est pas tout à fait le cas : le sujet de la forme causative doit être un nouvel argument qui vient s'ajouter à la proposition copulative de base, suite à l'introduction du verbe causatif. La relation entre la construction en avoir et la phrase spécificationnelle peut être qualifiée de reformulation thématique plutôt que de causativation. En effet, il semble possible de reformuler une phrase copulative spécificationnelle par une construction avec la séquence avoir...pour : il y a une relation de partie-tout très générale entre le sujet $\mathrm{Nb}$ et le $\mathrm{Na}$ sous pour de cette construction, qu'on retrouve exprimée de façon compacte dans une copulative spécificationnelle sous la forme du $S N$ complexe le $N a$ de $N b$. Les deux types de phrase sont en relation de paraphrase. On ne peut pas parler d'une causativation.

La deuxième hypothèse a ensuite été explorée. D'un côté, du fait du trait formel qui distingue (dans la plupart des cas) une phrase spécificationnelle des autres, c'est-à-dire la 
possibilité de former une phrase interrogative avec le mot interrogatif quel, et de l'autre, du fait que les propositions interrogatives indirectes partielles (IIP) sont toujours réductibles à une IIP en Quel est le $N$, on a supposé que les verbes recteurs d'une IIP pourraient être considérés comme des verbes causatifs d'une phrase spécificationnelle, impliquant toujours l'existence de cette dernière dans le procès décrit par le verbe principal, mais dont le contenu d'assertion n'est pas rendu explicite.

Avec les mêmes types de verbes, la contrepartie non interrogative (donc la prédication est explicite) semble se réaliser au moyen d'une subordonnée participiale introduite par comme étant. Dans ce cas-là, l'attribut n'est pas intégré dans la structure de phrase en tant qu'actant du verbe principal. La variante causative où il est syntaxiquement intégré, c'est-à-dire introduit simplement par comme, est possible, mais seulement si l'ordre des constituants de la phrase spécificationnelle s'inverse, en devenant, par le même coup, une prédication identificationnelle.

\section{Conclusions et perspective}

Dans la première partie de cette étude, trois types de constructions du verbe faire ont été distingués, malgré la forme de surface identique. Parmi ces trois types, il en a été idntifié un qui n'a pas fait l'objet d'une description approfondie dans la littérature, c'est la construction à attribut de l'objet indirect. En effet, l'aspect le plus important qui s'est dégagé au cours de la différenciation fonctionnelle en tro is types est que malgré sa position et sa construction, un type de $S N$ direct ne présente pas certaines propriétés fondamentales de l'objet direct et qu'il peut en revanche présenter quelques propriétés de l'attribut. Par l'interprétation non restreinte du sujet et par la paraphrase possible avec une construction factitive en $N$ faire $Q u P$, la construction en question peut être qualifiée de cons truction causative-attributive.

Dans la seconde partie, la construction à attribut de l'objet indirect a été mieux caractérisée, à l'aide de la typologie des phrases copulatives. Il en résulte qu'entre le complément indirect et son attribut, on n'observe qu'un type particulier de prédication, c'està-dire le type identificationnel. Par ailleurs, les opérateurs causatifs rendre et mettre s'avèrent être des opérateurs qui s'appliquent eux aussi à deux types particuliers de phrases copulatives, le type prédicationnel dont l'attribut est réalisé par l'adjectif et par le syntagme prépositionnel, respectivement. De ce point de vue, le verbe faire dans la construction à attribut peut être aligné à ces deux verbes, en tant qu'opérateur causatif qui s'applique, lui, à une phrase copulative identificationnelle.

Dans la littérature, la description complète de la distribution des formes causatives correspondant à tous les types de phrases copulatives ne semble pas avoir été faite. Cet examen a donc été entamé ici. Il en résulte que le verbe faire peut entrer dans deux formes de causatives de copulatives identificationnelles, selon que les attributs se réalisent par un nom sans déterminant $(N$ faire $N N$ ), soit par un nom avec déterminant ( $N$ faire de $N N$ ).

Pour ce qui concerne les cases vides dans la distribution (les formes causatives de : (a) une copulative prédicative dont l'attribut se réalise par un nom sans déterminant; (b) une copulative identificationnelle dont l'attribut se réalise par un nom sans déterminant; (c) une 
copulative spécificationnelle), quelques hypothèses ont été avancées pour les remplir, mais il faut conduire des études plus approfondies.

\section{Références bibliographiques}

Blanche-Benveniste, Claire. 1991. "Deux relations de solidarité utiles pour l'analyse de l'attribut." In $A$ la recherche de l'attribut, dir. par Marie-Madelaine De Gaulmyn et Sylvianne Rémi-Giraud, 83-97. Lyon : Presses Universitaires de Lyon.

Furukawa, Naoyo. 1987. "Sylvie a les yeux bleus : construction à double thème." Lingvisticae Investigationes $11: 283-302$.

Giry-Schneider, Jacqueline. 1978a. Les nominalisations en français : l'opérateur faire dans le lexique. Genève-Paris : Droz.

Giry-Schneider, Jacqueline. 1978b. "Interprétation aspectuelle des constructions verbales à double analyse." Lingvistica Investigationes 2 :23-53.

Giry-Schneider, Jacqueline. 1987. Les prédicats nominaux en français : les phrases simples à verbe support. Genève-Paris : Droz.

Gross, Maurice. 1976. "Sur quelques groupes nominaux complexes." In Méthodes en grammaire française, éds. par Jean-Claude Chevalier et Maurice Gross, 97-119. Paris : Klincksieck.

Gross, Maurice. 1981. “Les bases empiriques de la notion de prédicat sémantique.” Langages $63: 8-52$.

Grevisse, Maurice et André Goosse. 2011. Le Bon Usage (15 ${ }^{\text {ème }}$ édition). Bruxelles : De Boeck-Duculot.

Herslund, Michael. 1988a. Le datif en français. Leuven : Peeters.

Herslund, Michael. 1988b. "On Valence and Grammatical Relations." In Valency. Three Studies on the Linking Power of Verbs. Copenhagen Studies in Language (CEBAL Series 11), éd. par Finn Sørensen, 3-34. Copenhague : Hndelshøjskolens Forlag.

Herslund, Michael. 1994. "Valence et relations grammaticales." In Mélanges Lucien Tesnière. Linguistica XXXIV (1) : 109-117.

Herslund, Michael. 2004. "Valence, prédicat, préposition et la notion d'adjet." In La préposition en français II. Modèles linguistiques 54, XXVII-2, éds. par Danielle Leeman et Cécile Vaguer, 15-24, Toulon : Editions des Dauphins.

Herslund, Michael et Finn Sørensen. 1994. "A valence based theory of grammatical relations." In Function and Expression in Functional Grammar. éds. par Elisabeth EngbergPedersen et alii, 81-95. Berlin : Mouton de Gruyter.

Higgins, Francis Roger. 1973. The Pseudo-Cleft Construction in English. Thèse de Ph.D. Massachusetts Institute of Technology.

Kayne, Richard S. 1977. Syntaxe du français. Le cycle transformationnel. Paris : Seuil. 
Kupferman, Lucien. 2000. "Avoir et la predication seconde." Langue française 127 : 67-85.

Lauwers, Peter. 2008. "Les emplois attributifs de faire." Studia Neophilologica 80 : 43-64.

Muller, Claude. 1989. "Sur la syntaxe et la sémantique des relatives indépendantes et des interrogatives indirectes partielles." Revue Romane 24 :13-48.

Muller, Claude. 1996. La subordination en français : le schème corrélatif. Paris : Armand Colin.

Muller, Claude. 2000. 'Les constructions à adjectif attribut de l'objet, entre prédication seconde et complémentation verbale." Langue française 127 : 21-35.

Nakamura, Takuya. 2008. "La structure des interrogatives partielles directes et indirectes." Lingvistica Investigationes 31 (2) : 273-284.

Nilson-Ehle, Hans. 1953. "L'attribut de l'objet en français. Esquisse d'une étude." Studia Neophilologica 25 (4) : 105-140.

Olsson-Jonasson, Kerstin. 1981. "Thème, rhème, focus et la construction avec attribut de l'objet." Lingvistica Investigationes 5 (1) : 137-168.

Riegel, Martin. 1974. "L'adjectif attribut du complément d'objet direct : définition formelleet analyse sémantique." Travaux de linguistique et de littérature XII (1) : 229-248.

Riegel, Martin. 1985. L'adjectif attributif. Paris : P.U.F.

Riegel, Martin. 1988. "L'adjectif attribut de l'objet du verbe avoir : amalgame et prédication complexe." Travaux de linguistique 17 : 69-87.

Riegel, Martin. 1991. "Pour ou contre la notion grammaticale d'attribut de l'objet : critères et arguments." In $A$ la recherche de l'attribut, dir. par Marie-Madelaine De Gaulmyn et Sylvianne Rémi-Giraud, 99-118. Lyon : Presses Universitaires de Lyon.

Riegel, Martin. 1998. 'De il a les yeux bleus à je n'ai pas le cinq-à-sept facile : les avatars de la construction attributive de avoir." Travaux et recherches en linguistique appliquée (série B) 1 : 99-108.

Riegel, Martin. 2001. "Quelques remarques sur les constructions à attribut de l'objet issues d'une complétive attributive." In Langage et référence, mélanges offerts à K. Jonasson.

Studia Romanica Upsaliensia 63, éds. par Hans Kronning et alii, 543-553. Uppsala, Université d'Uppsala.

Riegel, Martin, Jean-Christophe Pellat, et René Rioul. 2009. Grammaire méthodique du français ( $4^{\mathrm{ème}}$ édition). Paris : P.U.F.

Tsuruga, Yoichiro. 2010. "La construction à attribut de l'objet direct en français : le cas du verbe juger (en japonais)." Area and Culture Studies 81, 339-382. Tokyo: Tokyo University of Foreign Studies.

Tsuruga, Yoichiro. 2013. "L'attribut et l'objet direct en français (en japonais).” In Furansugo wo toraeru. Tokyo : Sanshû-sha.

Van Peteghem, Marleen. 1987. "La réversibilité et les phrases copulatives spécificationnelles." Travaux de linguistiques 14/15 : 193-207.

Van Peteghem, Marleen. 1991. Les phrases copulatives dans les langues romanes. Wilhelmsfeld : G. Egert.

Wagner, Robert-Léon et Jacqueline Pinchon. 1962. Grammaire du français classique et moderne. Paris : Hachette. 
1 Désormais, nous entendons par "type A", "type B" et "type C", "phrases du type A, du type B et du type C", respectivement.

2 Voir, pour un exposé général, Gross (1976) et Giry-Schneider (1978a et 1978b).

3 Riegel et alii. (2009, 297).

4 Luc n'a pas un ami est possible. Dans ce cas-là, un ami est "le résultat d'une évaluation quantitative" (Riegel et alii., 2009, 297), ouvrant "la voie à des rectifications" (ibid.) comme Luc n'a pas un ami mais il en a plusieurs ou Luc n'a aucun ami.

5 Un verbe distributionnel est en opposition à un verbe support : il est distingué de ce dernier par sa capacité d'imposer sa propre grille d'arguments dans la proposition.

6 Pour l'aperçu général sur les constructions à verbe support, voir Gross (1981). Pour ce qui concerne les constructions syntaxiques autour du verbe support faire, voir Giry-Schneider (1978a, 1987).

7 Tsuruga $(2010,362)$ mentionne l'existence de la fonction d'attribut indirect d'un complément d'objet indirect à propos d'une construction du verbe juger: Peut-être certains (...) jugeront du tout comme peu novateur ; d'autres (...) le jugeront visionnaire (Swartz, B.). En effet, dans la première phrase, l'attribut du complément indirect en de est introduit indirectement par comme.

8 Wagner et Pinchon $(1962, \S 56)$, en parlant de l'attribut d'un complément, mentionne que dans l'exemple suivant le substantif attribut se rapporte à un complément déterminatif : J'ai fait de ma chambre la cellule d'un cloûtre. (A. De Vigny). D'après la terminologie de leur grammaire, un complément déterminatif s'oppose à la fois à l'attribut et à l'objet direct. Sous cette dénomination, les auteurs regroupent un argument du verbe construit indirectement (abuser du tabac, se moquer des règlements), aussi bien qu'un complément accessoire (abaisser le niveau de quelques degrés).

9 Voici la description de la fonction grammaticale Adjet par Herslund $(1994,113)$ :

Il n'y a en effet qu'une situation où le verbe peut spécifier un troisième actant : c'est la possibilité qu'ont certains verbes d'établir une relation de nature prédicative entre un de leurs actants et ce troisième actant. C'est la relation que j'appellerai adjet (A). Cette relation remplace un grand nombre de fonctions assez hétérogènes de la grammaire traditionnelle : l'objet indirect, l'objet prépositionnel, le complément local, le complément d'attribution, l'attribut du sujet et l'attribut de l'objet. (L'emphase est de Herslund.)

10 Lauwers (2008) est une rare étude consacrée à l'emploi attributif du verbe faire, mais dans son emploi copulatif. Cet auteur mentione la construction faire $+d e S N+S N$ (faire un salon d'une salle à manger), mais pour l'écarter tout de suite de son objet d'étude (les constructions intransitives).

11 Une autre série de phrases causatives avec le verbe faire qui met en œuvre l'infinitif à la place d'une complétive ne peut pas se mettre en parallèle avec la construction du type B. Sur ce point, voir la section 3.3.

12 La typologie de Higgins comporte le quatrième type que Van Peteghem a exclu de son étude : c'est le type que Higgins appelle phrase d'identité

13 Selon Van Peteghem $(1987,1991)$, le sujet et l'attribut (de surface) d'une phrase spécificationnelle sont l'attribut antéposé et le sujet postposé, respectivement. Dans cet article, quand nous parlons simplement du sujet et de l'attribut d'une phrase spécificationnelle, il s'agit respectivement du sujet et de l'attribut de surface.

14 La délimitation "dans cette construction" est importante puisque ce verbe ne refuse pas de se construire avec un adjectif ou un nom sans déterminant :

Il fait (beau, sombre, clair...)

Luc s'est fait (petit, beau,...)

Luc fait très (prof, médecin, artiste...)

Ce sont autant de constructions syntaxiques différentes que d'emplois différents du verbe faire.

15 Riegel $(2001,549-550)$ constate également la complémentarité de la distribution de ces deux types formels d'attribut.

16 Le verbe faire de l'exemp le Luc fait très (enfant, prof, étudiant, etc.) se trouve aussi dans ce paradigme. Pour cet emp loi copulatif du verbe faire, voir Lau wers (2008).

17 La structure $\mathrm{Nb}$ avoir pour $\mathrm{Na} \mathrm{Nc}$ présente donc une ambiguité au niveau de l'identification de l'attribut. Grevisse et Goosse (2011, §299), par exemple, considère que c'est le $\mathrm{Na}$ sous pour qui fonctionne comme attribut de $N c$ :

Avoir, suivi d'un attribut de l'objet (introduit par comme ou pour) peut recevoir comme objet direct un infinitif introduit par de ou une proposition [sans doute à la suite d'une ellipse ou d'une sorte d'haplologie (\$219) : Avoir pour mission de = avoir pour mission la mission de] : 
Il y a des hommes n'ayant pour mission [...] que de servir d'intermédiaires (Flaub., Educ., II,

4) (...).

Dans cette optique, la relation qu'a la phrase en avoir avec la phrase copulative n'est pas prise en compte. Soit dit en passant, l'analyse par ellipse que propose cette grammaire ne marche pas dans tous les cas, par exemple, la phrase Georges a eu pour amante Sylvie, qui doit recevoir, selon nous, la mê me analyse que l'exemple du Bon Usage, n'a pas pour paraphrase la phrase Georges a eu pour amante l'amante de Sylvie, qui ne veut pas dire du tout la même chose.

18 Selon Van Peteghem (1991), c'est en réalité le sujet de la phrase. 